\title{
Focus on the Synthesis and Reactions of Some New Pyridine Carbonitrile Derivatives as Antimicrobial and Antioxidant Agents
}

\author{
H.H. Sayed, E.M. Flefel, A.M. Abd El-Fattah*, W.I. \\ El-Sofany and A.M. Hassan ${ }^{* *}$ \\ Photochemistry Department, National Research Centre, and \\ *Chemistry Department, Faculty of Science, Cairo University, \\ Giza and ${ }^{* *}$ Department of Natural and Microbial Products \\ National Research Centre, Giza, Egypt.
}

\begin{abstract}
2-(4-CHLOROPHENYL)-1-(2,4-dichlorophenyl)-propen-1-one (1) $\checkmark$ was prepared and reacted with active methylene compound, ethyl cyanoacetate in the presence of ammonium acetate to give the corresponding pyridine carbonitrile (2). The behavior of compound 2 towards phosphorous pentasulfide, phosphorous oxychloride and some acyclic-sugars has been investigated and afforded compounds 3 , 4 and 5a-d, respectively. The thioxo-pyridine carbonitrile (3) reacted with different halo compounds namely: methyl iodide, ethyl chloroacetate, some acyclic sugars to afford 6,7 and $8 \mathrm{a}-\mathrm{c}$, respectively. Treatment of compound 3 with acrylonitrile afforded compound 9. Reaction of the thiosulfanyl 6 with hydrazine hydrate gave the hydrazino derivative 10 while reaction of 7 with the same reagents gave the acid hydrazide 11 . Also, compound 4 reacted with different nucleophiles to afford compounds 10, 12-14. Condensation of compound 10 with ethyl acetoacetate, acetyl acetone, acetic anhydride, p-chlorobenzaldehyde afforded compounds 15-18, respectively. Moreover, compound 10 reacted with carbon disulfide to afford compound 19. Finally, condensation of compound 10 with aldehydo-sugar namely: D-glucose gave the corresponding acyclic nucleoside 20. Furthermore, biological evaluation of some prepared compounds has been assessed and some of them revealed promising antimicrobial and antioxidant activity.
\end{abstract}

Keywords: Pyridines, Nicotinonitrile, Pyrazolopyridine, Triazolopyridine, Acyclic nucleosides, Antimicrobial activity and Antioxidant agent.

In a previous work, we reported that certain of our newly substituted pyridine derivatives exhibited anticonvulsant, analgesic, antiparkinsonian ${ }^{(1)}$ and antimicrobial $^{(2,3)}$ activities. Also, pyridine moiety showed antiplasmodial activity ${ }^{(4)}$, anticancer activities ${ }^{(5)}$, anti-infammatory ${ }^{(6)}$, antioxidant ${ }^{(7)}$ and anti-HIV activity ${ }^{(8)}$. Pyridinones were used for the treatment of ischemic stroke ${ }^{(9)}$. It displayed good antibiotic $^{(10)}$, antiparasite ${ }^{(11)}$, and antiviral potency against HIV $-1^{(12)}$. Some of triazolopyridine derivatives have inhibitory activity towards adenosine receptor ${ }^{(13)}$, antifungal activity ${ }^{(14)}$ and CNS depressing activity ${ }^{(15)}$. On the other hand pyrazolopyridine derivatives have oxidative hair dye properties ${ }^{(16)}$, antiviral activity $^{(17-19)}$, anti-inflamatory ${ }^{(20)}$, prevent renal ischemia reperfusion injury mice 
and they were used for detecting neuroinflammation in the brain ${ }^{(21)}$ such as Alzheimer disease. In view of these observations and in continuation of our previous work, we synthesized some new pyridine moiety and tested their biological activities. In general, compounds 3, 17 showed more antibacterial and antifungal activity than standard (drugs), while compound 20 showed high scavenging activity against artificial radicals.

\section{Results and Discussion}

2,4-Dichloroacetophenone reacted with p-chlorobenzaldehyde in ethanolic sodium hydroxide solution to produce 3-(4-chloro-phenyl)-1-(2,4-dichlorophenyl)-propen-1-one $(1)^{(22)}$. Analytical and spectral data of compound 1 are in total agreement with the proposed structure (c.f. Experimental Section). Condensation of chalcone 1 with ethyl cyanoacetate in the presence of ammonium acetate gave pyridine-3-carbonitrile (2). Also, compound 2 can be obtained in high yield, in one step synthesis, ${ }^{(23)}$ by heating a mixture of 2,4 -dichloroacetophenone, p-chlorobenzaldehyde and ethyl cyanoacetate in the presence of ammonium acetate (c.f. Experimental Section, Scheme 1). The structure of compound 2 was confirmed via spectral data. IR spectrum of 2 showed absorption bands at $\left(v, \mathrm{~cm}^{-1}\right): 2223$ $(\mathrm{CN})$ and $1656(\mathrm{CO}) .{ }^{1} \mathrm{H}$ NMR spectrum of compound 2 showed signals at $(\delta$ ppm): 6.36 (s, $1 \mathrm{H}$, pyridine $\mathrm{H}-5)$ and $13.00\left(\mathrm{~s}, 1 \mathrm{H}, \mathrm{NH}, \mathrm{D}_{2} \mathrm{O}\right.$ exchangeable). The mass spectrum showed $\mathrm{m} / \mathrm{z} 376(100 \%)$ supporting its molecular formula. compound 2 was thiated by using phosphorous pentasulfide in dry pyridine to afford thioxopyridine-3-carbonitrile derivative 3 . The structure of compound 3 was confirmed via spectral data. IR spectrum of 3 revealed the disappearance of $(\mathrm{CO})$ group as well as the existence of (CS) group at $1228 \mathrm{~cm}^{-1}$. The mass spectrum gave $\mathrm{m} / \mathrm{z} 392(100 \%)$. Compound 2 was chlorinated by using phosphorous oxychloride and phosphorous pentachloride to afford chloronicotinonitrile (4), the IR spectrum of 4 showed the absence of (CO) group,while its mass spectrum showed molecular ion peak at m/z 394 (100\%) (c.f. Experimental Section).

Also, when the sodium salt of compound 2 reacted with different cyclic or acyclic oxygenated alkyl halides gave analogous series of $\mathrm{N}$-acyclic nucleosides of pyridine derivatives 5a-d. The IR spectra revealed that, the sites of attack on compounds $5 \mathrm{a}-\mathrm{d}$ were at $\mathrm{N}$ - and not $\mathrm{O}$-atom ${ }^{(24)}$ due to the presence of absorption bands at $\left(\mathrm{v}, \mathrm{cm}^{-1}\right) 1650(\mathrm{CO}) .{ }^{1} \mathrm{H}$ NMR spectrum of compound $5 \mathrm{c}$, as a representative example, showed signals at $(\delta \mathrm{ppm}): 3.35\left(\mathrm{~s}, 3 \mathrm{H}, \mathrm{OCH}_{3}\right)$, $3.40\left(\mathrm{t}, 2 \mathrm{H}, \mathrm{CH}_{2} \mathrm{~N}\right)$ and $3.60\left(\mathrm{t}, 2 \mathrm{H}, \mathrm{CH}_{2} \mathrm{O}\right)$ ( Scheme 1).

When the thioxocarbonitrile 3 reacted with methyl iodide in alcoholic potassium hydroxide or ethyl chloroacetate in the presence of sodium ethoxide, it gave the corresponding methylsulfanyl derivative 6 or sulfanyl ethyl ester derivative 7 , respectively. Also, when the sodium salt of compound 3 was treated with some cyclic or acyclic oxygenated alkyl halides it afforded the corresponding oxyalkylsulfanyl derivatives $8 \mathrm{a}-\mathrm{c}$, respectively. The IR spectra revealed that, the sites of the attack were at $\mathrm{S}$ - and not $\mathrm{N}$-atom due to the absence of bands characteristic for $(\mathrm{NH})$ and $(\mathrm{CS})$ groups ${ }^{(25)}$. ${ }^{1} \mathrm{HNMR}$ spectrum of compound 8c, as a representative example, signals at $(\delta \mathrm{ppm}): 3.00\left(\mathrm{t}, 2 \mathrm{H}, \mathrm{CH}_{2}\right)$, $3.30\left(\mathrm{~s}, 3 \mathrm{H}, \mathrm{CH}_{3}\right)$ and $3.80\left(\mathrm{t}, 2 \mathrm{H}, \mathrm{CH}_{2}\right)$ ( Scheme 2).

Egypt. J. Chem. 53, No.1 (2010) 


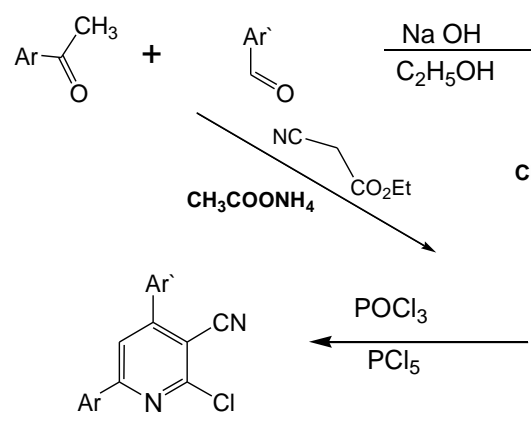<smiles>CCOC(=O)CC#N</smiles><smiles>N#Cc1c(Br)cc([Al])[nH]c1=S</smiles>

2

3

DMF, $\mathrm{NaH}$<smiles>[R]n1c(Br)cc(Br)c(C#N)c1=O</smiles>

$a: R=\searrow$

$\mathbf{b}: \mathrm{R}=\widehat{\mathrm{OH}}$

$c: R=\curvearrowright O$

$\mathbf{d}: \mathrm{R}=\stackrel{\mathrm{O}}{\mathrm{N}}$

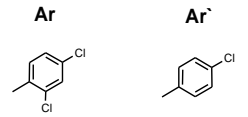

Scheme 1

In the meantime, when 3 was treated with acrylonitrile in pyridine it gave the corresponding sulfanylpropionamide derivative 9. When compound 4 and also 6 were treated with hydrazine hydrate, the same hydrazino derivative 10 was obtained in $64 \%$ and $85 \%$ yield, respectively (Schemes $2 \& 3$ ). The structure of 10 was confirmed by spectral data. The IR spectrum of 10 showed the absence of (CO) of ester and showed absorption bands at 3423, 3141, 2218 characteristic for $\mathrm{NH}_{2}, \mathrm{NH}, \mathrm{CN}$ groups, respectively. ${ }^{1} \mathrm{H}$ NMR spectrum (DMSO- $\mathrm{d}_{6}$ ) of compound 10 showed the expected signals corresponding to the aromatic protons and two types of exchangeable protons corresponding to $\mathrm{NH}, \mathrm{NH}_{2}$ groups. Its mass spectrum showed m/z 390.00 (100\%) supporting its molecular formula. 


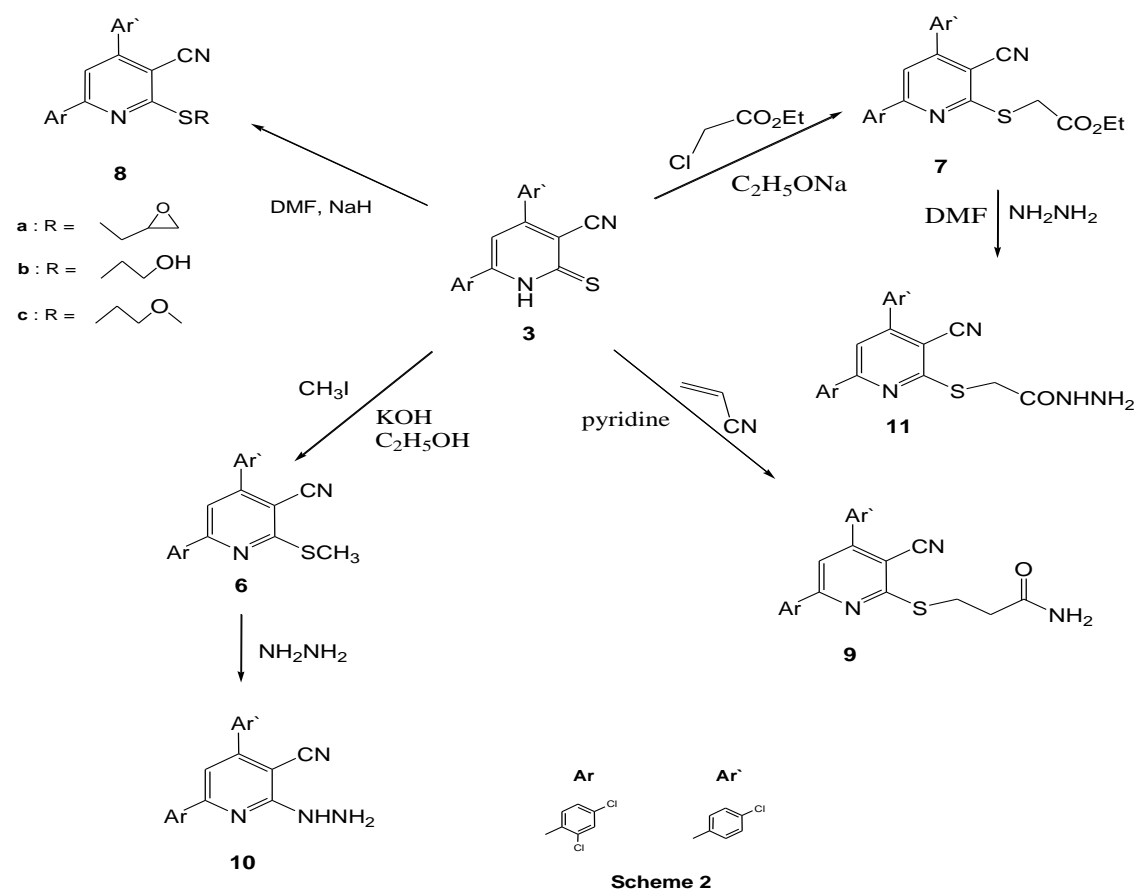

Scheme 2
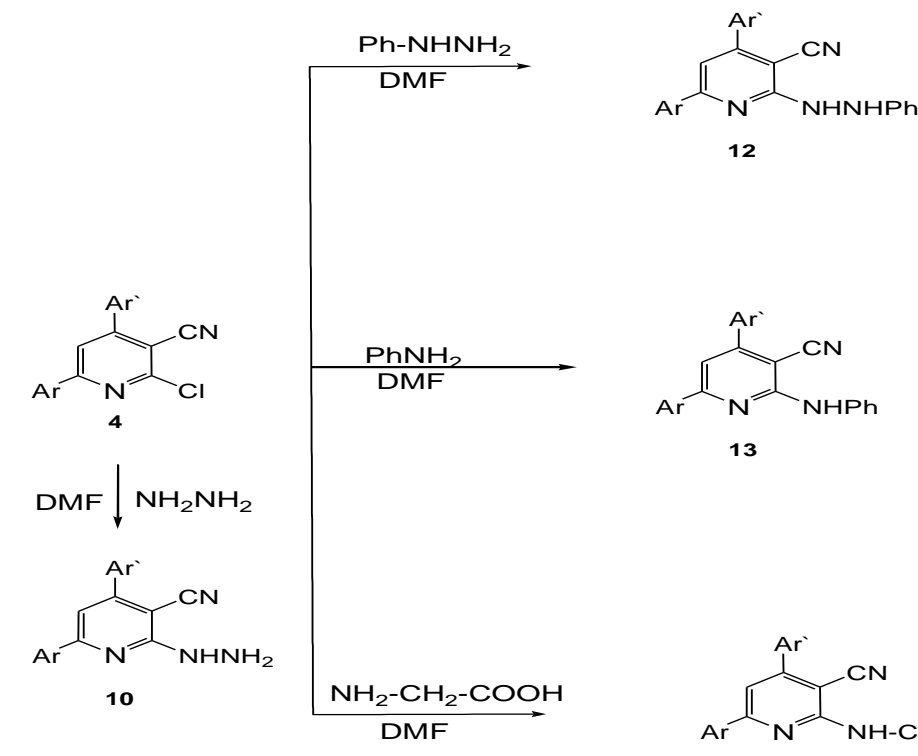

ar

Scheme 3
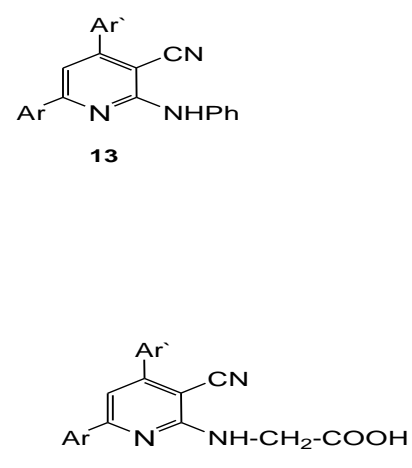

14

Scheme 3

Egypt. J. Chem. 53, No.1 (2010) 
Reaction of compound 7 with hydrazine hydrate gave the acid hydrazide (11) (Scheme 2). The structure of compound 11 was confirmed with spectral data.The IR spectrum of compound 11 showed absorption bands at $\left(v, \mathrm{~cm}^{-1}\right): 3428-3350$ $\left(\mathrm{NH}_{2}\right), 3166(\mathrm{NH}), 1679(\mathrm{CO})$ characteristic for amide groups. ${ }^{1} \mathrm{H} \mathrm{NMR}$ spectrum (DMSO-d $)_{6}$ ) showed signals at $3.70\left(\mathrm{~s}, 2 \mathrm{H}, \mathrm{CH}_{2}\right), 4.20\left(\mathrm{~s}, 2 \mathrm{H}, \mathrm{NH}_{2}, \mathrm{D}_{2} \mathrm{O}\right.$ exchangeable), and $9.80\left(\mathrm{~s}, 1 \mathrm{H}, \mathrm{NH}, \mathrm{D}_{2} \mathrm{O}\right.$ exchangeable). Its mass spectrum showed m/z 464 (7\%) (c.f. Experimental Section).

Compound 4 reacted with different nucleophiles namely phenyl hydrazine, aniline or glycine in DMF to afford compounds 12-14, respectively (c.f. Experimental Section, Scheme 3).

The 2-hydrazino-nicotinonitrile (10) is another key compound which enriches the synthesis of the heterocyclic compounds. It reacted with different $\beta$ diketones, namely: ethyl acetoacetate, acetyl acetone to afford 15 and 16, respectively (c.f. Experimental Section, Scheme 4). Also, on heating compound 10 with acetic anhydride, it afforded triazolopyridine (17) (Scheme 4). In addition, compound 10 was condensed with 4-chlorobenzaldehyde or D-glucose to yield 18 or 20 , respectively. Also, compound 10 was treated with carbon disulfide in sodium ethoxide solution to afford compound 19 (Scheme 4).
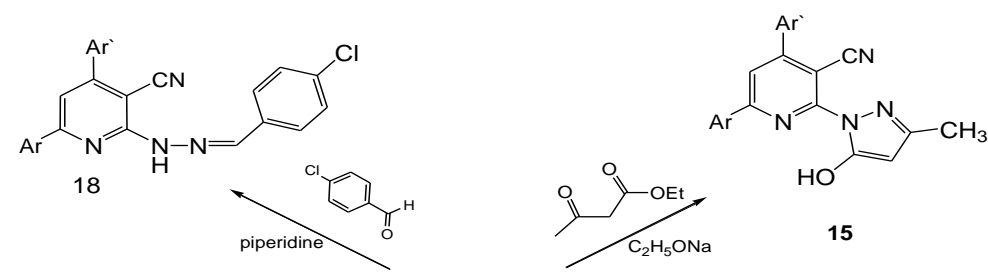
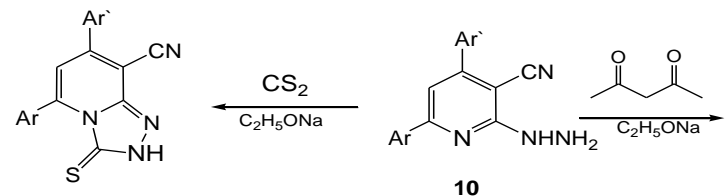

10

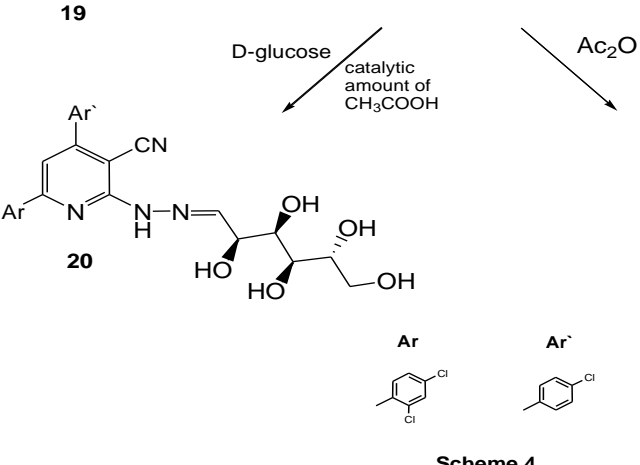

17

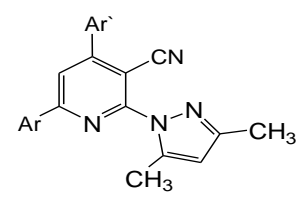

16<smiles>CC1NN=C2C(C#N)=C(Br)C=C(Cl)N21</smiles>

Scheme 4 


\section{Antimicrobial Activity}

Some of the synthesized compounds $1-3,9,10,15,17$ and 18-20 were tested in concentration of $0.1 \mathrm{~g} / \mathrm{ml}$ using dimethylformamide as a solvent.

\section{Microorganisms'species}

Bacteria : a) Gram-negative bacteria, Eschericha coli., Salmonella typhi

b) Gram-positive bacteria, Bacillus subtitles, Staphylococcus aureus, Streptococcus

Yeast: Candida albicans, Sacchromyces

Fungi: Aspergillus niger.

\section{Medium}

The cap-assay method containing (g/l): peptone 6.0, yeast extract 3.0, meat extract 1.5, glucose 1.0 and agar 20.0 were used. The medium was sterilized and divided while hot $\left(50-60^{\circ} \mathrm{C}\right)$ in $15 \mathrm{ml}$. Portions among sterile Petri-dishes of $9 \mathrm{~cm}$ diameter.

One $\mathrm{ml}$ of the spor suspension of each microorganism was spread all over the surface of the cold solid medium placed in the Petri-dish.

\section{Method $^{(26)}$}

$0.5 \mathrm{~g}$ of each of the tested compounds was dissolved in $5 \mathrm{ml}$ of dimethylformamide. An amount of $0.1 \mathrm{ml}$ of test solution was placed on Watman paper disc of $9 \mathrm{~mm}$ diameter and the solvent was left to evaporate. These saturated discs were placed carefully on the surface of the inoculated solid medium; each Petri-dish contains at least 3 discs. The Petri-dishes were incubated at $5^{\circ} \mathrm{C}$ for an hour to permit good diffusion and then transferred to an incubator of $85^{\circ} \mathrm{C}$ overnight then examined. The results were then recorded by measuring the inhibition zone diameters.

Among the tested compounds, it was noticed that compounds 3 and 17 showed more significant antibacterial and antifungal activity than some known drugs (standers). The results of antimicrobial screening were recorded as average diameter of inhibition zone in $\mathrm{mm}$ and presented in Table 1. 
TABLE 1. Antimicrobial activities of the tested compounds.

\begin{tabular}{|c|c|c|c|c|c|c|c|c|}
\hline \multirow{5}{*}{$\begin{array}{c}\text { Tested } \\
\text { Compounds } \\
\& \\
\text { Standers } \\
\end{array}$} & \multicolumn{8}{|c|}{ Inhibition Zone (mm) } \\
\hline & \multicolumn{8}{|c|}{ Microorganism } \\
\hline & \multicolumn{5}{|c|}{ Bacteria } & \multirow{3}{*}{$\begin{array}{c}\text { Fungi } \\
\begin{array}{c}A . \\
\text { niger }\end{array}\end{array}$} & \multirow{2}{*}{\multicolumn{2}{|c|}{ Yeast }} \\
\hline & \multicolumn{2}{|c|}{ Gram-negative } & \multicolumn{3}{|c|}{ Gram-positive } & & & \\
\hline & $\begin{array}{c}E . \\
\text { coli. }\end{array}$ & $\begin{array}{c}S . \\
\text { typhi }\end{array}$ & $\begin{array}{c}B . \\
\text { subtitls }\end{array}$ & $\begin{array}{c}S . \\
\text { aureus }\end{array}$ & $\begin{array}{l}\text { Strepto } \\
\text { coccus }\end{array}$ & & $\begin{array}{c}C . \\
\text { albicans }\end{array}$ & $\begin{array}{c}\text { Sacchro } \\
\text { myces }\end{array}$ \\
\hline Control: DMSO & - & - & - & - & - & - & - & - \\
\hline Streptomycin & +++ & +++ & +++ & +++ & +++ & + & +++ & +++ \\
\hline Erythromycin & + & - & +++ & ++ & ++ & - & - & - \\
\hline Fusidic Acid & - & - & - & - & - & +++ & +++ & +++ \\
\hline 1 & + & - & + & - & - & + & + & - \\
\hline 2 & + & + & - & - & - & - & + & - \\
\hline 3 & +++ & + & +++ & ++ & ++ & + & +++ & ++ \\
\hline 9 & ++ & ++ & + & ++ & ++ & + & - & ++ \\
\hline 10 & - & - & - & - & - & - & - & - \\
\hline 15 & + & + & + & ++ & + & + & + & + \\
\hline 17 & ++ & ++ & ++ & +++ & ++ & + & + & ++ \\
\hline 18 & + & + & + & ++ & + & + & + & ++ \\
\hline 19 & + & + & + & ++ & + & + & + & + \\
\hline 20 & + & - & + & - & - & - & - & - \\
\hline
\end{tabular}

+++ Highly sensitive (inhibition zone $=21-40 \mathrm{~mm}$ )

++ Fairly sensitive (inhibition zone $=16-20 \mathrm{~mm}$ ).

+ Slightly sensitive (inhibition zone $=10-15 \mathrm{~mm}$ ).

- Not sensitive.

\section{Antioxidant Activity}

\section{Material and methods}

Free radical scavenging activity (in vitro antioxidant)

The scavenging of DPPH radical was carried out according to the described $\operatorname{method}^{(27)}$. One $\mathrm{ml}$ of various concentrations of the each compound in methanol and $5 \mathrm{ml}$ of freshly prepared $0.1 \mathrm{MM}$ methanol solution of DPPH were thoroughly mixed, and kept for $50 \mathrm{~min}$ in the dark. The absorbance of the reaction mixture at $517 \mathrm{~nm}$ was read with a spectrophotometer (UV- 1600 Shimazdu) against a blank. The percentage of free radical scavenging was calculated as follows:

$$
\text { Scavenging effect }=\left(1-\left(\mathrm{A}_{517 \mathrm{~nm}, \text { sample }} / \mathrm{A}_{517 \mathrm{~nm} \text {, blank }}\right)\right) \times 100
$$

Extract conc. providing 50\% inhibition (IC50) was calculated from the graph plotted free radical scavenging percentage against extract conc., tests were carried out in triplicate ${ }^{(28)}$.

\section{Results and discussion}

Reactive species that initiate reactions which damage organic molecules of biological importance, are considered to be the cause of several health problems including cancer, heart diseases and possibly, the process of aging itself ${ }^{(28)}$. Also, lipid oxidation by radical results in food deterioration, especially in high fat 
foods ${ }^{(29)}$. Synthetic antioxidants such as butylated hydroxyanisole (BHA) and butylated hydroxytoluene (BHT) are used as antioxidants. However, demand for natural antioxidants has been increased due to consumer concerns about the safety of synthetic antioxidants. Antioxidants, either as additives or as pharmaceutical supplements, can terminate radical reactions in vivo ${ }^{(30)}$.

Many researchers have focused on natural antioxidants such as ascorbic acid and -tocopherol rather than synthetic antioxidants such as butylated hydroxyanisole (BHA) and butylated hydroxytoluene (BHT). Synthetic antioxidants have been widely used in the food industry for extending the shelf life of foods that are susceptible to lipid oxidation. However, there are some arguments about the safety and adverse effects of synthetic antioxidants as food additives ${ }^{(31)}$.

\section{Conclusion}

Some new pyridinone derivatives, hydrazinopyridine $5 b, c, 10$, oxyalkylsulfanyl pyridine derivatives $(8 \mathrm{~b}, \mathrm{c})$ pyrazole $(15)$ and hexylidinonecotinonitrile (20) were prepared and were selected for screening of antioxidant activity.

Compound 20 exhibited a high scavenging activity against the artificial radical DPPH when compared to the other compounds. From the chemical point of view, compound 20 containing hydroxyl groups which are responsible for antioxidant potential of compound.

TABLE 2. Free radical scavenging capacities of the compounds measured in DPPH assay.

\begin{tabular}{|c|c|}
\hline Compound & IC $_{\mathbf{5 0}} \quad(\boldsymbol{\mu m o l e} / \mathbf{m l})$ \\
\hline $5 \mathrm{~b}$ & $>1000$ \\
\hline $5 \mathrm{c}$ & $>1000$ \\
\hline 10 & $>1000$ \\
\hline $8 \mathrm{~b}$ & $>1000$ \\
\hline $8 \mathrm{c}$ & $>1000$ \\
\hline 15 & $>1000$ \\
\hline 20 & $<1000$ \\
\hline
\end{tabular}
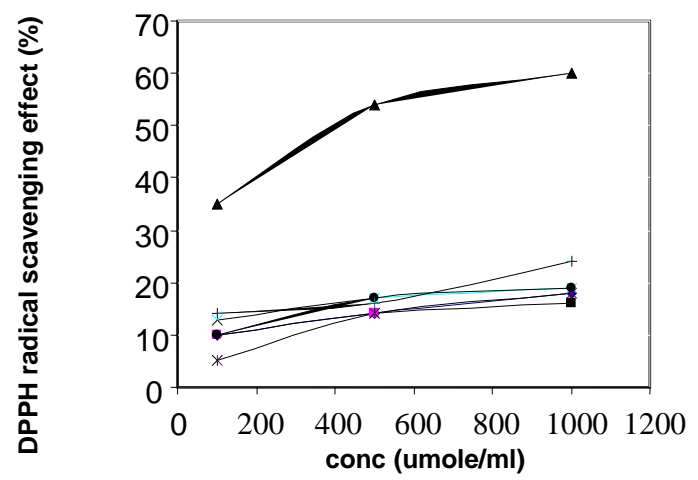

\section{$-15$}

$-5 \mathrm{c}$

$-20$

$\times 8 \mathrm{c}$

* $5 \mathrm{~b}$

$-8 \mathrm{~b}$

$+10$

Fig. 1. DPPH free radical scavenging effect of compounds. Values are mean of triplicates . 


\section{Experimental}

Melting points were measured using Electrothermal IA 9100 apparatus, (Shimadzu, Tokyo, Japan). IR spectra were recorded as potassium bromide pellets on a Perkin-Elmer 1650 spectrophotometer (Perkin-Elmer, Norwalk, CT, USA). ${ }^{1} \mathrm{H}$ NMR was determined on a Jeol-Ex-300 NMR spectrometer (JEOL, Tokyo, Japan) and chemical shifts were expressed as part per million; ppm $(\delta$ values) against TMS as internal reference. Mass spectra were recorded on VG 2AM-3F mass spectrometer (Thermo electron corporation, USA) and exhibited the pattern of the isotope abundance of chlorine atoms ${ }^{(32)}$ which is given for compound 1 as a representative example. Microanalyses were operated using Mario El Mentar apparatus, Organic Microanalysis Unit. Follow up of the reactions and checking the purity of the compounds was made by TLC on silica gel-precoted aluminum sheets (Type 60 F254, Merck, and Darmstadt, Germany).

3-(4-Chlorophenyl)-1-(2,4-dichlorophenyl)-propen-1-one (1)

A mixture of 2,4-dichloroacetophenone $1(1.88 \mathrm{gm}, 0.01 \mathrm{~mole})$ and $\mathrm{p}$ chlorobenzaldehyde (1.41 gm, 0.01 mole) in $30 \mathrm{ml}$ ethanol and $15 \mathrm{ml}$ of $10 \%$ $\mathrm{NaOH}$ was heated and stirred for $3 \mathrm{hr}$. After cooling, the reaction mixture was poured onto ice and the obtained precipitate was filtered off, dried and recrystallized from ethanol to give compound 1, 75\% yield; m.p. $150-151^{\circ} \mathrm{C}$; IR spectrum $\left(\mathrm{KBr}, v, \mathrm{~cm}^{-1}\right): 1660(\mathrm{CO}), 1606(\mathrm{CC}) ;{ }^{1} \mathrm{H}$ NMR (DMSO-d 6 ,

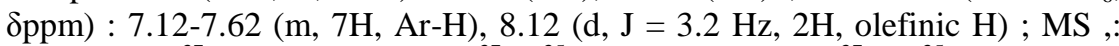
$\mathrm{m} / \mathrm{z}:\left(\mathrm{M}^{+}, 3 \mathrm{Cl}^{37}, 316,1 \%\right), \quad\left(\mathrm{M}^{+}, 2 \mathrm{Cl}^{37}, \mathrm{Cl}^{35}, 314,18 \%\right), \quad\left(\mathrm{M}^{+}, \mathrm{Cl}^{37}, 2 \mathrm{Cl}^{35}, 312,60 \%\right)$, $\left(\mathrm{M}^{+}, 3 \mathrm{Cl}^{35}, 310,62 \%\right)$. Analysis for $\mathrm{C}_{15} \mathrm{H}_{9} \mathrm{Cl}_{3} \mathrm{O}(310)$ : required $\mathrm{C}, 57.82 ; \mathrm{H}$, $2.91 ; \mathrm{Cl}, 34.13$; found $\mathrm{C}, 57.92 ; \mathrm{H}, 2.80 ; \mathrm{Cl}, 34.35$.

\section{4-(4-Chlorophenyl)-6-(2,4-dichlorophenyl)-2-oxo-1,2-dihydropyridine-3-carbonitrile (2) Method A}

A mixture of 1 (3.12 gm, 0.01 mole), ethyl cyanoacetate (1.13 gm, 0.01 mole) and ammonium acetate (6.16 gm, 0.08 mole) in $40 \mathrm{ml}$-butanol was refluxed for $10 \mathrm{hr}$. The precipitate was filtered off, dried and recrystallized from dioxane to give compound $2,30 \%$ yield.

\section{Method B}

A mixture of 2,4-dichloroacetophenone (1.88 gm, 0.01 mole $), \quad p$ chlorobenzaldehyde (1.41 gm, 0.01 mole), ethyl cyanoacetate (1.13 gm, 0.01 mole) and ammonium acetate $(6.16 \mathrm{gm}, 0.08$ mole) in $40 \mathrm{ml}$-butanol was refluxed for $3 \mathrm{hr}$, yellow crystals were obtained, then filtered off, washed with water, dried and recrystallized to give compound $2,80 \%$ yield; mp $279-280^{\circ} \mathrm{C}$; IR spectrum (KBr, v, $\left.\mathrm{cm}^{-1}\right): 3444(\mathrm{NH}), 2223(\mathrm{CN}), 1656(\mathrm{CO}) \mathrm{cm}^{-1} ;{ }^{1} \mathrm{H}$ NMR spectrum (DMSO-d $\left.\mathrm{d}_{6}, \delta \mathrm{ppm}\right): 6.36(\mathrm{~s}, 1 \mathrm{H}$, pyridine $\mathrm{H}), 7.60-8.40(\mathrm{~m}, 7 \mathrm{H}, \mathrm{Ar}-\mathrm{H})$, 13.00 (s, 1H, NH, $\mathrm{D}_{2} \mathrm{O}$ exchangeable); $\mathrm{MS}, \mathrm{m} / \mathrm{z}(\%): 376 \quad\left(\mathrm{M}^{+}, 3 \mathrm{Cl}^{35}, 100\right)$. Analysis for $\mathrm{C}_{18} \mathrm{H}_{9} \mathrm{Cl}_{3} \mathrm{~N}_{2} \mathrm{O}$ (375.64): required $\mathrm{C}, 57.55 ; \mathrm{H}, 2.41 ; \mathrm{Cl}, 28.31 ; \mathrm{N}$, 7.46; found $\mathrm{C}, 57.64 ; \mathrm{H}, 2.44 ; \mathrm{Cl}, 28.44 ; \mathrm{N}, 7.53$ 
4-(4-Chlorophenyl)-6-(2,4-dichlorophenyl) -2-thioxo-1,2-dihydropyridine-3carbonitrile (3)

A mixture of $2(3.75 \mathrm{gm}, 0.01 \mathrm{~mole})$ and $\mathrm{P}_{2} \mathrm{~S}_{5}(2.22 \mathrm{gm}, 0.01 \mathrm{~mole})$ in $20 \mathrm{ml}$ dry pyridine was refluxed for $5 \mathrm{hr}$. After cooling, the reaction mixture was poured into ice, the separated solid was filtered off, dried and recrystallized from methanol to give compound 3,74\% yield; m.p. $265-266^{\circ} \mathrm{C}$; IR spectrum $(\mathrm{KBr}, \mathrm{v}$, $\left.\mathrm{cm}^{-1}\right): 3425(\mathrm{NH}), 2220(\mathrm{CN}), 1228(\mathrm{CS}) ;{ }^{1} \mathrm{H}$ NMR spectrum (DMSO-d ${ }_{6}, \delta$ ppm): $6.60(\mathrm{~s}, 1 \mathrm{H}$, pyridine $\mathrm{H}), 7.40-7.90(\mathrm{~m}, 7 \mathrm{H}, \mathrm{Ar}-\mathrm{H}), 12.55\left(\mathrm{~s}, 1 \mathrm{H}, \mathrm{NH}, \mathrm{D}_{2} \mathrm{O}\right.$ exchangeable); MS : $\mathrm{m} / \mathrm{z}(\%): 392\left(\mathrm{M}^{+}, 3 \mathrm{Cl}^{35}, 100\right)$. Analysis for $\mathrm{C}_{18} \mathrm{H}_{9} \mathrm{Cl}_{3} \mathrm{~N}_{2} \mathrm{~S}$ (391.70): required C, 55.19; H, 2.32; Cl, 27.15; N, 7.15; S, 8.19. Found C, 55.21; $\mathrm{H}, 2.29 ; \mathrm{Cl}, 27.05 ; \mathrm{N}, 7.22 ; \mathrm{S}, 8.18$.

2-Chloro-4-(4-chlorophenyl)-6-(2,4-dichlorophenyl) nicotinonitrile (4)

A suspension of $2(3.75 \mathrm{gm}, 0.01$ mole $)$ in $\mathrm{POCl}_{3}(4.6 \mathrm{ml}, 0.03 \mathrm{~mole})$ and (2.1 gm, 0.01 mole) of $\mathrm{PCl}_{5}$ was heated on steam bath for $2 \mathrm{hr}$. The reaction mixture was poured into crushed ice; the separated solid was filtered off and recrystallized from dioxane to give compound 4, $79 \%$ yield; m.p.289-290 ${ }^{\circ} \mathrm{C}$; IR spectrum (KBr, v, cm $\left.{ }^{-1}\right)$ : $2221(\mathrm{CN}) ;{ }^{\mathrm{H}} \mathrm{NMR}$ spectrum (DMSO-d $6 . \delta \mathrm{ppm}$ ): $6.62(\mathrm{~s}, 1 \mathrm{H}$, pyridine $\mathrm{H}), 7.62-8.31(\mathrm{~m}, 7 \mathrm{H}, \mathrm{Ar}-\mathrm{H}) ; \mathrm{MS} \mathrm{m} / \mathrm{z}(\%): 394\left(\mathrm{M}^{+}, 3 \mathrm{Cl}^{35}\right.$, 100). Analysis for $\mathrm{C}_{18} \mathrm{H}_{8} \mathrm{Cl}_{4} \mathrm{~N}_{2}$ (394): required $\mathrm{C}, 54.86 ; \mathrm{H}, 2.05 ; \mathrm{Cl}, 35.99 ; \mathrm{N}$, 7.11. Found C, 54.76; H, 2.25; Cl, 35.89; N, 7.07.

Synthesis of compounds 5 a-d

To a solution of $2(3.75 \mathrm{gm}, 0.01 \mathrm{~mole})$ in $20 \mathrm{ml}$ dry DMF, sodium hydride $\left(0.24 \mathrm{~g}, 0.01\right.$ mole) was added and the reaction mixture was stirred at $70^{\circ} \mathrm{C}$ for 3 hr. After cooling, epichlorohydrine, chloroethanol, 2-chloroethyl methyl ether or chloroacetate $(0.01$ mole) was added with stirring at at room temperature for $5 \mathrm{hr}$, evaporated under reduced pressure, the residue was solidified with water, filtered off and crystallized from ethanol to give compounds $5 \mathrm{a}-\mathrm{d}$.

4-(4-Chlorophenyl)-6-(2,4- dichlorophenyl)-1- oxiranylmethyl-2-oxo-1,2-dihydropyridine-3-carbonitrile $(5 a)$

$55 \%$ yield ; m.p.172-173 ${ }^{\circ} \mathrm{C}$; IR spectrum $\left(\mathrm{KBr}, v, \mathrm{~cm}^{-1}\right): 2222(\mathrm{CN}), 1658$ (CO); ${ }^{1} \mathrm{H}$ NMR spectrum (DMSO-d 6 , $\delta$ ppm): 2.5 (d, $\left.2 \mathrm{H}, \mathrm{J}=4.2 \mathrm{~Hz}, \mathrm{CH}_{2}\right), 3.09$ $\left(\mathrm{d}, 2 \mathrm{H}, \mathrm{J}=6.5 \mathrm{~Hz}, \mathrm{OCH}_{2}\right), 3.16(\mathrm{~m}, 1 \mathrm{H}, \mathrm{OCH}), 7.54-8.31(\mathrm{~m}, 8 \mathrm{H}, \mathrm{Ar}-\mathrm{H}+$ pyridine $\mathrm{H}) ; \mathrm{MS}: \mathrm{m} / \mathrm{z}(\%): 432\left(\mathrm{M}^{+}, 3 \mathrm{Cl}^{35}, 19\right)$. Analysis for $\mathrm{C}_{21} \mathrm{H}_{13} \mathrm{Cl}_{3} \mathrm{~N}_{2} \mathrm{O}_{2}$ (431.70): required $\mathrm{C}, 58.43 ; \mathrm{H}, 3.04 ; \mathrm{Cl}, 24.64 ; \mathrm{N}, 6.49$; found $\mathrm{C}, 58.51 ; \mathrm{H}, 3.09$; $\mathrm{Cl}, 24.62 ; \mathrm{N}, 6.57$.

4-(4-Chlorophenyl)-6-(2,4-dichlorophenyl)-1-(2-hydroxyethyl)-2-oxo -1, 2-dihydropyridine-3-carbonitrile $(5 b)$

$45 \%$ yield ; m.p. $151-152^{\circ} \mathrm{C}$; IR spectrum $\left(\mathrm{KBr}, v, \mathrm{~cm}^{-1}\right): 3383(\mathrm{OH}), 2223$

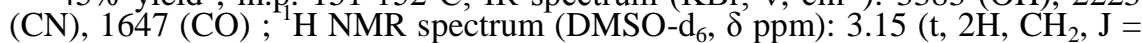
$2.4 \mathrm{~Hz}), 3.79\left(\mathrm{t}, 2 \mathrm{H}, \mathrm{OCH}_{2}, \mathrm{~J}=3.0 \mathrm{~Hz}\right), 4.4-4.45\left(\mathrm{br}, 1 \mathrm{H}, \mathrm{OH}, \mathrm{D}_{2} \mathrm{O}\right.$ exchangeable), 7.54-8.31 (m, 8H, Ar-H + pyridine $\mathrm{H}) ; \mathrm{MS}: \mathrm{m} / \mathrm{z}(\%): 420\left(\mathrm{M}^{+}\right.$, $\left.3 \mathrm{Cl}^{35}, 35\right)$. Analysis for $\mathrm{C}_{20} \mathrm{H}_{13} \mathrm{Cl}_{3} \mathrm{~N}_{2} \mathrm{O}_{2}(419.69)$ : required $\mathrm{C}, 57.24 ; \mathrm{H}, 3.12 ; \mathrm{Cl}$, $25.34 ; \mathrm{N}, 6.67$; found $\mathrm{C}, 57.29 ; \mathrm{H}, 3.14 ; \mathrm{Cl}, 25.30 ; \mathrm{N}, 6.62$.

4-(4-Chlorophenyl)-6-(2,4-dichlorophenyl)-1-(2-methoxy-ethyl)-2-oxo-1,2dihy-dropyridine-3-carbonitrile $(5 c)$

$50 \%$ yield ; m.p. $129-130^{\circ} \mathrm{C}$; IR spectrum $\left(\mathrm{KBr}, v, \mathrm{~cm}^{-1}\right): 2222(\mathrm{CN}), 1649$ (CO); ${ }^{1} \mathrm{H}$ NMR spectrum (DMSO-d $\left.{ }_{6}, \delta \mathrm{ppm}\right): 3.35\left(\mathrm{~s}, 3 \mathrm{H}, \mathrm{OCH}_{3}\right), 3.40(\mathrm{t}, 2 \mathrm{H}$, $\left.\mathrm{CH}_{2} \mathrm{~N}, \mathrm{~J}=2.5 \mathrm{~Hz}\right), 3.60\left(\mathrm{t}, 2 \mathrm{H}, \mathrm{CH}_{2} \mathrm{O}, \mathrm{J}=3.2 \mathrm{~Hz}\right) 6.40(\mathrm{~s}, 1 \mathrm{H}$, pyridine $\mathrm{H})$, $7.30-7.90(\mathrm{~m}, 7 \mathrm{H}$, Ar-H) ; MS :m/z $\%): 434\left(\mathrm{M}^{+}, 3 \mathrm{Cl}^{35}, 25\right)$. Analysis for $\mathrm{C}_{21} \mathrm{H}_{15} \mathrm{Cl}_{3} \mathrm{~N}_{2} \mathrm{O}_{2}(433.71)$ : required $\mathrm{C}, 58.15 ; \mathrm{H}, 3.49 ; \mathrm{Cl}, 24.52 ; \mathrm{N}, 6.46$; found $\mathrm{C}$, $58.25 ; \mathrm{H}, 3.51 ; \mathrm{Cl}, 24.48 ; \mathrm{N}, 6.49$. 
4-(4-Chlorophenyl)-6-(2,4-dichlorophenyl)-2-oxo-1-(2-oxo-propyl)-1, 2-dihydropyridine-3-carbonitrile (5d)

$62 \%$ yield $62 \%$; m.p. $210-211^{\circ} \mathrm{C}$; IR spectrum $\left(\mathrm{KBr}, v, \mathrm{~cm}^{-1}\right): 2218(\mathrm{CN})$, $1726(\mathrm{CO}), 1650(\mathrm{CO}) ;{ }^{1} \mathrm{H}$ NMR spectrum (DMSO-d 6 , $\delta \mathrm{ppm}$ ): 2.09 (s, $3 \mathrm{H}$, $\left.\mathrm{O}=\mathrm{C}-\mathrm{CH}_{3}\right), 4.12\left(\mathrm{~s}, 2 \mathrm{H}, \mathrm{CH}_{2} \mathrm{~N}\right), 6.40(\mathrm{~s}, 1 \mathrm{H}$, pyridine $\mathrm{H}), 7.30-7.90(\mathrm{~m}, 7 \mathrm{H} \mathrm{Ar}-$ H) ; MS : $\mathrm{m} / \mathrm{z}(\%): 432\left(\mathrm{M}^{+}, 3 \mathrm{Cl}^{35}, 27\right)$. Analysis for $\mathrm{C}_{21} \mathrm{H}_{13} \mathrm{Cl}_{3} \mathrm{~N}_{2} \mathrm{O}_{2}(431.70)$ : required $\mathrm{C}, 58.43 ; \mathrm{H}, 3.04 ; \mathrm{Cl}, 24.64 ; \mathrm{N}, 6.49$; found $\mathrm{C}, 58.49 ; \mathrm{H}, 3.01 ; \mathrm{Cl}$, $24.61 ; \mathrm{N}, 6.50$.

4-(4-Chlorophenyl)-6-(2,4-dichlorophenyl)-2-methylsulfanylnicotinonitrile (6)

A solution of 3 (3.92 gm, $0.01 \mathrm{~mole}$ ) in $50 \mathrm{ml}$ ethanolic potassium hydroxide $(5 \%)$ was heated for $30 \mathrm{~min}$ at $80^{\circ} \mathrm{C}$. After cooling at room temperature, methyl iodide ( $2.8 \mathrm{gm}, 0.12 \mathrm{~mole}$ ) was added to the reaction mixture, and then heated at $80{ }^{\circ} \mathrm{C}$ for $5 \mathrm{hr}$, poured into water. The separated solid was filtered off, washed with water, dried and recrystallized from methanol to give compound 6,70\% yield 70\%; m.p. $269-270^{\circ} \mathrm{C}$; IR spectrum $\left(\mathrm{KBr}, \mathrm{v}, \mathrm{cm}^{-1}\right): 2221(\mathrm{CN})$ and absence of $(\mathrm{NH}) ;{ }^{1} \mathrm{H}$ NMR spectrum (DMSO-d $\left.\mathrm{d}_{6}, \delta \mathrm{ppm}\right): 2.60\left(\mathrm{~s}, 3 \mathrm{H}, \mathrm{S}-\mathrm{CH}_{3}\right), 6.62(\mathrm{~s}$, $1 \mathrm{H}$, pyridine $\mathrm{H}), 7.30-7.50(\mathrm{~m}, 7 \mathrm{H}, \mathrm{Ar}-\mathrm{H})$; MS: $\mathrm{m} / \mathrm{z}(\%): 406\left(\mathrm{M}^{+}, 3 \mathrm{Cl}^{35}, 100\right)$. Analysis for $\mathrm{C}_{19} \mathrm{H}_{11} \mathrm{Cl}_{3} \mathrm{~N}_{2} \mathrm{~S}$ (405.73): required C, 56.25; H, 2.73; Cl, 26.21; N, 6.90; S, 7.90; found C, 56.29; H, 2.77; Cl, 26.33; N, 6.81; S, 7.80.

[4-(4-Chlorophenyl)-3-cyano-6-(2,4-dichlorophenyl)-pyridin-2-ylsulfanyl] acetic acid ethyl ester (7)

A solution of 3 (3.92 gm, 0.01 mole) in $30 \mathrm{ml}$ sodium ethoxide $(0.23 \mathrm{gm}$ sodium metal in $30 \mathrm{ml}$ ethanol) was heated for $30 \mathrm{~min}$ at $80{ }^{\circ} \mathrm{C}$. The reaction mixture was cooled, and then ethyl chloroacetate $(1.5 \mathrm{gm}, 0.012 \mathrm{~mole})$ was added with stirring at $70{ }^{\circ} \mathrm{C}$ for $3 \mathrm{hr}$, poured into water, the precipitated solid was filtered off, washed with water, dried and recrystallized from methanol to give compound 7, 66\% yield; m.p. $169-170^{\circ} \mathrm{C}$; IR spectrum $\left(\mathrm{KBr}, v, \mathrm{~cm}^{-1}\right): 2224$ $(\mathrm{CN}), 1741(\mathrm{CO}), 1546(\mathrm{CN}) ;{ }^{1} \mathrm{H}$ NMR spectrum (DMSO-d $\left.6, \delta \mathrm{ppm}\right): 1.30(\mathrm{t}$, $3 \mathrm{H}, \mathrm{CH}_{3}, \mathrm{~J}=3.4 \mathrm{~Hz}$ ), 3.90 (s, $2 \mathrm{H}, \mathrm{CH}_{2}$ ), 4.20 (q, $2 \mathrm{H}, \mathrm{CH}_{2}, \mathrm{~J}=4.5 \mathrm{~Hz}$ ), 7.20-7.90 $(\mathrm{m}, 8 \mathrm{H}, \mathrm{Ar}-\mathrm{H}+$ pyridine $\mathrm{H})$; $\mathrm{MS}: \mathrm{m} / \mathrm{z}(\%)$ : $478\left(\mathrm{M}^{+}, 3 \mathrm{Cl}^{35}, 9\right)$. Analysis for $\mathrm{C}_{22} \mathrm{H}_{15} \mathrm{Cl}_{3} \mathrm{~N}_{2} \mathrm{O}_{2} \mathrm{~S}$ (477.79): required $\mathrm{C}, 55.36 ; \mathrm{H}, 3.16 ; \mathrm{Cl}, 22.26 ; \mathrm{N}, 5.86 ; \mathrm{S}$, 6.71; found $\mathrm{C}, 55.39 ; \mathrm{H}, 3.26 ; \mathrm{Cl}, 22.36 ; \mathrm{N}, 5.88 ; \mathrm{S}, 6.71$. 
Synthesis of compounds $8 a-c$

To a solution of 3 (3.92 gm, 0.01 mole) in $20 \mathrm{ml}$ dry DMF, sodium hydride $(0.24 \mathrm{gm}, 0.01 \mathrm{~mole})$ was added. The reaction mixture was stirred at $70^{\circ} \mathrm{C}$ for $3 \mathrm{hr}$, cooled, and then epichlorohydrine, chloroethanol and/or 2-chloroethyl methyl $(0.01$ mole) was added, stirred at room temperature for $5 \mathrm{hr}$. The reaction mixture was evaporated under reduced pressure; the residue was washed with distilled water, filtered off and dried to give compounds 8a-c and crystallized from ethanol.

4-(4-Chlorophenyl)-6-(2,4-dichlorophenyl)-2-oxiranylmethylsulfanylnicotinonitrile(8a)

$65 \%$ yield ; m.p. $190-192^{\circ} \mathrm{C}$; IR spectrum $\left(\mathrm{KBr}, v, \mathrm{~cm}^{-1}\right): 2223(\mathrm{CN}), 1644$ (CO) ; ${ }^{1} \mathrm{H}$ NMR spectrum (DMSO-d $\left.\mathrm{d}_{6}, \delta \mathrm{ppm}\right): 2.40\left(\mathrm{~d}, 2 \mathrm{H}, \mathrm{J}=5.6 \mathrm{~Hz}, \mathrm{CH}_{2}\right.$ ), $2.70\left(\mathrm{~d}, 2 \mathrm{H}, \mathrm{CH}_{2}, \mathrm{~J}=4.6 \mathrm{~Hz}\right), 3.10(\mathrm{~m}, \mathrm{H}, \mathrm{CH}), 7.30-7.90(\mathrm{~m}, 8 \mathrm{H}, \mathrm{Ar}-\mathrm{H}+$ pyridine $\mathrm{H})$; MS: $\mathrm{m} / \mathrm{z}(\%)$ : $447\left(\mathrm{M}^{+}, 3 \mathrm{Cl}^{35}, 13\right)$. Analysis for $\mathrm{C}_{21} \mathrm{H}_{13} \mathrm{Cl}_{3} \mathrm{~N}_{2} \mathrm{OS}$ (447.76): required $\mathrm{C}, 56.33 ; \mathrm{H}, 2.93 ; \mathrm{Cl}, 23.75 ; \mathrm{N}, 6.26 ; \mathrm{S}, 7.16$; found $\mathrm{C}, 56.43$; $\mathrm{H}, 2.97 ; \mathrm{Cl}, 23.79 ; \mathrm{N}, 6.26 ; \mathrm{S}, 7.22$.

4-(4- Chlorophenyl)- 6- (2,4- dichlorophenyl)- 2 -(2-hydroxyethylsulfanyl) nicotinonitrile $(8 b)$

$57 \%$ yield; m.p. $148-150^{\circ} \mathrm{C}$; IR spectrum $\left(\mathrm{KBr}, \mathrm{v}, \mathrm{cm}^{-1}\right): 3400(\mathrm{OH}), 2226$ $(\mathrm{CN}) ;{ }^{1} \mathrm{H}$ NMR spectrum (DMSO-d $\left.{ }_{6}, \delta \mathrm{ppm}\right): 3.15\left(\mathrm{t}, 2 \mathrm{H}, \mathrm{CH}_{2} \mathrm{~J}=1.6 \mathrm{~Hz}\right), 3.79$ (m, $2 \mathrm{H}, \mathrm{CH}_{2}, \mathrm{~J}=2.4 \mathrm{~Hz}$ ), 4.4-4.6 (br, $1 \mathrm{H}, \mathrm{OH}, \mathrm{D}_{2} \mathrm{O}$ exchangeable), 6.50 (s, 1H, pyridine $\mathrm{H}), 7.54-8.31$ (m, 7H Ar-H) ; MS: m/z(\%): $436\left(\mathrm{M}^{+}, 3 \mathrm{Cl}^{35}, 8\right)$. Analysis for $\mathrm{C}_{20} \mathrm{H}_{13} \mathrm{Cl}_{3} \mathrm{~N}_{2} \mathrm{OS}$ (435.75): required $\mathrm{C}, 55.13 ; \mathrm{H}, 3.01 ; \mathrm{Cl}, 24.41 ; \mathrm{N}$, 6.43; S, 7.36; found C, 55.23; H, 3.09; Cl, 24.45; N, 6.43; S, 7.47.

4-(4- Chlorophenyl)- 6-(2,4-dichlorophenyl)- 2-(2-methoxyethylsulfanyl) nicotinonitrile $(8 c)$

$67 \%$ yield; m.p. $253-254^{\circ} \mathrm{C}$; IR spectrum $\left(\mathrm{KBr}, v, \mathrm{~cm}^{-1}\right): 2222(\mathrm{CN}) ;{ }^{1} \mathrm{H}$ NMR spectrum (DMSO-d 6 , $\delta$ ppm): $3.00\left(\mathrm{t}, 2 \mathrm{H}, \mathrm{N}-\mathrm{CH}_{2}\right), 3.30\left(\mathrm{~s}, 3 \mathrm{H}, \mathrm{O}-\mathrm{CH}_{3}\right)$, 3.80 (t, $\left.2 \mathrm{H}, \mathrm{O}-\mathrm{CH}_{2}, \mathrm{~J}=2.6 \mathrm{~Hz}\right), 6.40$ (s, 1H, pyridine $\left.\mathrm{H}\right), 7.30-7.90(\mathrm{~m}, 7 \mathrm{H} \mathrm{Ar}-\mathrm{H})$; MS: $\mathrm{m} / \mathrm{z}(\%): 450\left(\mathrm{M}^{+}, 3 \mathrm{Cl}^{35}, 8\right)$. Analysis for $\mathrm{C}_{21} \mathrm{H}_{15} \mathrm{Cl}_{3} \mathrm{~N}_{2} \mathrm{OS}(449.78)$ : required C, 56.08; H, 3.36; Cl, 23.65; N, 6.23; S, 7.13; found C, 56.28; H, 3.38; Cl, 23.67; N, 6.20; S, 7.19.

N-[4-(4-Chlorophenyl)-3-cyano-6-(2,4-dichlorophenyl) pyridin- 2- yl-sulfanyl] propionamide (9)

A mixture of 3 (3.92 gm, 0.01 mole) and acrylonitrile ( $0.53 \mathrm{gm}, 0.01 \mathrm{~mole}$ ) in $20 \mathrm{ml}$ dry pyridine was refluxed for $4 \mathrm{hr}$. After cooling, the reaction mixture poured into ice, the formed solid was filtered off and recrystallized from methanol to give compound 9 in $65 \%$ yield ; m.p. $300^{\circ} \mathrm{C}$; IR spectrum $(\mathrm{KBr}, v$, $\left.\mathrm{cm}^{-1}\right)$ : 3400, $3390\left(\mathrm{NH}_{2}\right), 2226(\mathrm{CN}), 1790(\mathrm{CO}) ;{ }^{1} \mathrm{H}$ NMR spectrum (DMSO-d 6 , $\delta$ ppm): $3.2\left(\mathrm{t}, 2 \mathrm{H}, \mathrm{CH}_{2}, \mathrm{~J}=2.6 \mathrm{~Hz}\right), 3.60\left(\mathrm{t}, 2 \mathrm{H}, \mathrm{CH}_{2}, \mathrm{~J}=2.8 \mathrm{~Hz}\right), 4.4(\mathrm{~s}, 2 \mathrm{H}$, $\mathrm{NH}_{2}, \mathrm{D}_{2} \mathrm{O}$ exchangeable), $6.26(\mathrm{~s}, 1 \mathrm{H}$, pyridine $\mathrm{H}), 7.63-7.90(\mathrm{~m}, 7 \mathrm{H}, \mathrm{Ar}-\mathrm{H})$; MS : m/z(\%) : $463\left(\mathrm{M}^{+}, 3 \mathrm{Cl}^{35}, 30\right)$. Analysis for $\mathrm{C}_{21} \mathrm{H}_{14} \mathrm{Cl}_{3} \mathrm{~N}_{3} \mathrm{OS}$ (462.78):

Egypt. J. Chem. 53, No.1 (2010) 
required $\mathrm{C}, 54.50 ; \mathrm{H}, 3.05 ; \mathrm{Cl}, 22.98 ; \mathrm{N}, 9.08 ; \mathrm{S}, 6.93$; found $\mathrm{C}, 54.58 ; \mathrm{H}, 3.09$; $\mathrm{Cl}, 23.01 ; \mathrm{N}, 9.10 ; \mathrm{S}, 6.89$.

\section{4-(4-Chlorophenyl)-6-(2,4-dichlorophenyl)-2-hydrazinonicotinonitrile (10) \\ Method A}

A solution of compound 6 ( $3.9 \mathrm{gm}, 0.01 \mathrm{~mole})$ in DMF $(20 \mathrm{ml})$, hydrazine hydrate $(0.64 \mathrm{gm}, 0.02)$ was added. The solution mixture was refluxed for $6 \mathrm{hr}$. Upon cooling, the formed precipitate was filtered off and recrystallized from DMF to give compound 10 in yield 64\%; m.p.270-272 ${ }^{\circ}$.

\section{Method B}

A solution of compound 4 (4.0gm, $0.01 \mathrm{~mole})$ in DMF (30 ml), hydrazine hydrate $(0.64 \mathrm{gm}, 0.02)$ was added and the reaction mixture was refluxed for $6 \mathrm{hr}$. The separated solid after cooling was recrystallized from DMF to give compound 10 in yield $85 \%$; m.p. $270-272^{\circ} \mathrm{C}$; IR spectrum $\left(\mathrm{KBr}, v, \mathrm{~cm}^{-1}\right): 3423$ $\left(\mathrm{NH}_{2}\right), 3141(\mathrm{NH}), 2218(\mathrm{CN}) \mathrm{cm}^{-1} ;{ }^{1} \mathrm{H}$ NMR spectrum (DMSO-d $\left.{ }_{6}, \delta \mathrm{ppm}\right): 4.40$ (s, $2 \mathrm{H}, \mathrm{NH}_{2}, \mathrm{D}_{2} \mathrm{O}$ exchangeable), 7.54-8.31 (m, $8 \mathrm{H}, \mathrm{Ar}-\mathrm{H}+$ pyridine $\left.\mathrm{H}\right), 9.6$ (s, $1 \mathrm{H}, \mathrm{NH}, \mathrm{D}_{2} \mathrm{O}$ exchangeable); MS: $\mathrm{m} / \mathrm{z}(\%): 390\left(\mathrm{M}^{+}, 3 \mathrm{Cl}^{35}, 100\right)$. Analysis for $\mathrm{C}_{18} \mathrm{H}_{11} \mathrm{Cl}_{3} \mathrm{~N}_{4}$ (389.66): required C, 55.48; $\mathrm{H}, 2.85 ; \mathrm{Cl}, 27.29 ; \mathrm{N}, 14.38$; found $\mathrm{C}$, 55.49; H, 2.84; Cl, 27.34; N, 14.30.

[4-(4-Chlorophenyl)-3-cyano-6-(2,4-dichlorophenyl) pyridin-2-ylsulfanyl] acetic acid hydrazide (11)

A mixture of 7 (4.78 gm, 0.01 mole) and hydrazine hydrate (0.64 gm, 0.02 mole $)$ in dioxane $(30 \mathrm{ml})$ was refluxed for $8 \mathrm{hr}$. The separated solid was recrystallized from dioxane to give compound 11 in $80 \%$ yield ; m.p. 298$300^{\circ} \mathrm{C}$; IR spectrum $\left(\mathrm{KBr}, \mathrm{v}, \mathrm{cm}^{-1}\right): 3428,3350\left(\mathrm{NH}_{2}\right), 3166(\mathrm{NH}), 2217(\mathrm{CN})$, 1679 (CO) ; ${ }^{1} \mathrm{H}$ NMR spectrum (DMSO-d 6 , $\delta$ ppm): $3.70\left(\mathrm{~s}, 2 \mathrm{H}, \mathrm{CH}_{2}\right.$ ), 4.20 (s, $2 \mathrm{H}, \mathrm{NH}_{2}, \mathrm{D}_{2} \mathrm{O}$ exchangeable), 7.63-7.85 (m, $8 \mathrm{H}, \mathrm{Ar}-\mathrm{H}+$ pyridine $\left.\mathrm{H}\right)$, and 9.80 (s, H, NH, $\mathrm{D}_{2} \mathrm{O}$ exchangeable) ; $\mathrm{MS}: \mathrm{m} / \mathrm{z}(\%)$ : $464\left(\mathrm{M}^{+}, 3 \mathrm{Cl}^{35}, 7\right)$. Analysis for $\mathrm{C}_{20} \mathrm{H}_{13} \mathrm{Cl}_{3} \mathrm{~N}_{4} \mathrm{OS}$ (463.77): required $\mathrm{C}, 51.80 ; \mathrm{H}, 2.83 ; \mathrm{Cl}, 22.93 ; \mathrm{N}, 12.08 ; \mathrm{S}$, 6.91; found C, 51.66; H, 2.83; Cl, 22.89; N, 12.15; S, 6.98 .

Synthesis of compounds 12-14

A mixture of 4 (3.96 gm, 0.01 mole) and phenyl hydrazine, aniline or glycine (0.01 mole) in $20 \mathrm{ml} \mathrm{DMF}$ was refluxed for $6 \mathrm{hr}$. After cooling, the reaction mixture was poured into cold water; the formed solid was filtered off and recrystallized from the proper solvent to give compounds 12-14,respectively.

4-(4-Chlorophenyl)-6-(2,4-dichlorophenyl)-2-(2-phenylhydrazino) nicotinonitrile

It recrystallized from dioxane $65 \%$ yield ; m.p. $134-136^{\circ} \mathrm{C}$; IR spectrum $\left(\mathrm{KBr}, v, \mathrm{~cm}^{-1}\right): 3160(\mathrm{NH}), 3210(\mathrm{NH}), 2210(\mathrm{CN}) ;{ }^{1} \mathrm{H}$ NMR spectrum (DMSO$\left.\mathrm{d}_{6}, \delta \mathrm{ppm}\right):$ 7.53-8.32 (m, 13H, Ar-H + pyridine $\left.\mathrm{H}\right), 8.90\left(\mathrm{~s}, \mathrm{H}, \mathrm{NH}, \mathrm{D}_{2} \mathrm{O}\right.$ exchangeable), 9.60 (s, H, NH, $\mathrm{D}_{2} \mathrm{O}$ exchangeable) ; $\mathrm{MS}: \mathrm{m} / \mathrm{z}(\%): 467\left(\mathrm{M}^{+}\right.$, 
$\left.3 \mathrm{Cl}^{35}, 25\right)$. Analysis for $\mathrm{C}_{24} \mathrm{H}_{15} \mathrm{Cl}_{3} \mathrm{~N}_{4}$ (466.76): required $\mathrm{C}, 61.89 ; \mathrm{H}, 3.25 ; \mathrm{Cl}$, 22.84; N, 12.03; found C, 61.83; H, 3.21; Cl, 22.82; N, 12.04 .

4-(4-Chlorophenyl)-6-(2,4-dichloro-phenyl)-2-(phenylamino) nicotinonitrile (13)

It recrystallized from ethyl acetate $68 \%$ yield ; m.p. $177-179^{\circ} \mathrm{C}$; IR spectrum $\left(\mathrm{KBr}, v, \mathrm{~cm}^{-1}\right): 3174(\mathrm{NH}), 2210(\mathrm{CN}) ;{ }^{1} \mathrm{H}$ NMR spectrum (DMSO-d $\left.\mathrm{d}_{6}, \delta \mathrm{ppm}\right)$ : 7.45-8.55 (m, 13H, Ar-H + pyridine $\mathrm{H}), 9.40$ (s, H, NH, $\mathrm{D}_{2} \mathrm{O}$ exchangeable) ; MS : m/z(\%):451 $\left(\mathrm{M}^{+}, 3 \mathrm{Cl}^{35}, 8\right)$. Analysis for $\mathrm{C}_{24} \mathrm{H}_{14} \mathrm{Cl}_{3} \mathrm{~N}_{3}$ (450.75): required $\mathrm{C}$, 63.95; H, 3.13; Cl, 23.60; N, 9.32; found C, 64.01; H, 3.18; Cl, 23.69; N, 9.30.

[4-(4-Chlorophenyl) -3- cyano -6-(2,4- dichlorophenyl)- pyridin -2-ylamino] acetic acid (14)

It recrystallized from ethanol $72 \%$ yield; m.p. $162-164{ }^{\circ} \mathrm{C}$; IR spectrum $\left(\mathrm{KBr}, v, \mathrm{~cm}^{-1}\right): 3450(\mathrm{OH}), 3157(\mathrm{NH}), 2210(\mathrm{CN}), 1703(\mathrm{CO}) ;{ }^{1} \mathrm{H}$ NMR spectrum (DMSO-d $\left.{ }_{6}, \delta \mathrm{ppm}\right): 3.35\left(\mathrm{~s}, 2 \mathrm{H}, \mathrm{CH}_{2}\right), 4.7\left(\mathrm{~s}, 1 \mathrm{H}, \mathrm{OH}, \mathrm{D}_{2} \mathrm{O}\right.$ exchangeable), 7.00-7.40 (m, $8 \mathrm{H}, \mathrm{Ar}-\mathrm{H}+$ pyridine $\mathrm{H}), 8.40\left(\mathrm{~s}, 1 \mathrm{H}, \mathrm{NH}, \mathrm{D}_{2} \mathrm{O}\right.$ exchangeable); MS : m/z(\%): $433\left(\mathrm{M}^{+}, 3 \mathrm{Cl}^{35}, 6\right)$. Analysis for $\mathrm{C}_{20} \mathrm{H}_{12} \mathrm{Cl}_{3} \mathrm{~N}_{3} \mathrm{O}_{2}$ (432.69): required $\mathrm{C}, 55.52 ; \mathrm{H}, 2.80 ; \mathrm{Cl}, 24.58 ; \mathrm{N}, 9.71$; found $\mathrm{C}, 55.60 ; \mathrm{H}, 2.75$; $\mathrm{Cl}, 24.62 ; \mathrm{N}, 9.68$.

\section{Synthesis of compounds 15 and 16}

To a warmed sodium ethoxide solution $(0.23 \mathrm{gm}$ sodium metal in $30 \mathrm{ml}$ ethanol), $10\left(3.9 \mathrm{gm}, 0.01\right.$ mole) was added and heated at $80^{\circ} \mathrm{C}$ for $30 \mathrm{~min}$. After cooling, ethyl acetoacetate or acetyl acetone (0.012 mole) was added to the reaction mixture, and then for $3 \mathrm{hr}$, poured into water. The formed solid was filtered off and recrystallized from the proper solvent to give compounds 15 and 16.

4-(4-Chlorophenyl)-6-(2,4-dichlorophenyl)-2-(3-methyl-5-hydroxypyrazol-1y) nicotinonitrile (15)

It recrystallized from methanol $70 \%$ yield ; m.p. $174-176^{\circ} \mathrm{C}$; IR spectrum $\left(\mathrm{KBr}, v, \mathrm{~cm}^{-1}\right): 3854(\mathrm{NH}), 2212(\mathrm{CN}), 1690(\mathrm{CO}) ;{ }^{1} \mathrm{H}$ NMR spectrum (DMSO$\left.\mathrm{d}_{6}, \delta \mathrm{ppm}\right): 1.80\left(\mathrm{~s}, 3 \mathrm{H}, \mathrm{CH}_{3}\right), 4.96(\mathrm{~s}, 1 \mathrm{H}$, pyrazole $\mathrm{H}), 7.0\left(\mathrm{~s}, 1 \mathrm{H}, \mathrm{OH}, \mathrm{D}_{2} \mathrm{O}\right.$ exchangeable), 7.04 (s, 1H, pyridine $\mathrm{H}), 7.37-7.58$ (m, 7H Ar-H); MS : m/z (\%):456 $\left(\mathrm{M}^{+}, 3 \mathrm{Cl}^{35}, 16\right)$. Analysis for $\mathrm{C}_{22} \mathrm{H}_{13} \mathrm{Cl}_{3} \mathrm{~N}_{4} \mathrm{O}$ (455.72): required $\mathrm{C}, 57.98$; $\mathrm{H}, 2.88 ; \mathrm{Cl}, 23.34 ; \mathrm{N}, 12.29$; found $\mathrm{C}, 58.03 ; \mathrm{H}, 2.96 ; \mathrm{Cl}, 23.41 ; \mathrm{N}, 12.39$.

4-(4- Chlorophenyl)- 6-(2, 4- dichlorophenyl) -2-(3,5- dimethylpyrazol-1-yl) nicotinonitrile (16)

It recrystallized from ethanol ; $69 \%$ yield; m.p. $175-177^{\circ} \mathrm{C}$; IR spectrum $\left(\mathrm{KBr}, v, \mathrm{~cm}^{-1}\right): 2226(\mathrm{CN}) ;{ }^{1} \mathrm{H}$ NMR spectrum (DMSO-d $\left.{ }_{6}, \delta \mathrm{ppm}\right): 2.90(\mathrm{~s}, 6 \mathrm{H}$, $\left.2 \mathrm{CH}_{3}\right), 5.10(\mathrm{~s}, 1 \mathrm{H}$, pyrazole $\mathrm{H}), 6.20(\mathrm{~s}, 1 \mathrm{H}$, pyridine $\mathrm{H}), 7.30-8.00(\mathrm{~m}, 7 \mathrm{H}, \mathrm{Ar}-$ H) ppm; MS: m/z(\%): $454\left(\mathrm{M}^{+}, 3 \mathrm{Cl}^{35}, 100\right)$. Analysis for $\mathrm{C}_{23} \mathrm{H}_{15} \mathrm{Cl}_{3} \mathrm{~N}_{4}(453.75)$ : required $\mathrm{C}, 60.88 ; \mathrm{H}, 3.33 ; \mathrm{Cl}, 23.44 ; \mathrm{N}, 12.35$; found $\mathrm{C}, 60.68 ; \mathrm{H}, 3.34 ; \mathrm{Cl}$, $23.54 ; \mathrm{N}, 12.37$. 
7-(4-Chloro-phenyl)-5-(2,4-dichloro-phenyl)-3-methyl-3,8a-dihydro-[1,2,4] triazolo [4,3-a]pyridine-8-carbonitrile (17)

A solution of $10(3.9 \mathrm{gm} .0 .01 \mathrm{~mole})$ in $10 \mathrm{ml}$ acetic anhydride was refluxed for $2 \mathrm{hr}$. After cooling, the reaction mixture was poured into water; the separated solid was collected by filtration and recrystallized from acetic acid to give compound $17,61 \%$ yield ; m.p. $156-157^{\circ} \mathrm{C}$; IR spectrum $\left(\mathrm{KBr}, v, \mathrm{~cm}^{-1}\right): 3235$ $(\mathrm{NH}), 2224(\mathrm{CN}) ;{ }^{1} \mathrm{H}$ NMR spectrum (DMSO-d 6 , $\left.\delta \mathrm{ppm}\right): 1.70\left(\mathrm{~s}, 3 \mathrm{H}, \mathrm{CH}_{3}\right)$, $2.90(\mathrm{~s}, 1 \mathrm{H}$, triazole $\mathrm{H}), 7.20-7.90(\mathrm{~m}, 8 \mathrm{H}, \mathrm{Ar}-\mathrm{H}+$ pyridine $\mathrm{H})$, and $10.80(\mathrm{~s}, 1 \mathrm{H}$, $\mathrm{NH}, \mathrm{D}_{2} \mathrm{O}$ exchangeable) ; MS: $\mathrm{m} / \mathrm{z}(\%): 416\left(\mathrm{M}^{+}, 3 \mathrm{Cl}^{35}, 65\right)$. Analysis for $\mathrm{C}_{20} \mathrm{H}_{13} \mathrm{Cl}_{3} \mathrm{~N}_{4}$ (415.70): required $\mathrm{C}, 57.79 ; \mathrm{H}, 3.15 ; \mathrm{Cl}, 25.59 ; \mathrm{N}, 13.48$; found $\mathrm{C}$, $57.73 ; \mathrm{H}, 3.09 ; \mathrm{Cl}, 25.61 ; \mathrm{N}, 13.50$.

2-[N'-(4-Chloro-benzylidene)-hydrazino]-4-(4-chloro-phenyl)-6-(2,4-dichloro-phenyl)nicotinonitrile (18)

To a solution of $10(3.9 \mathrm{gm}, 0.01 \mathrm{~mole})$ in $20 \mathrm{ml}$ DMF in the presence of $0.1 \mathrm{ml}$ of piperidine, p-chlorobenzaldehyde ( $1.41 \mathrm{gm}, 0.01 \mathrm{~mole})$ was added. The reaction mixture was refluxed for $5 \mathrm{hr}$, the formed solid was filtered off and recrystallized from acetic acid to give compound $18,79 \%$ yield; m.p. $320-322^{\circ} \mathrm{C}$; IR spectrum $\left(\mathrm{KBr}, v, \mathrm{~cm}^{-1}\right): 3140(\mathrm{NH}), 2217(\mathrm{CN}) ;{ }^{1} \mathrm{H}$ NMR spectrum (DMSO-d $\left.\mathrm{d}_{6}, \delta \mathrm{ppm}\right): 7.20-7.90(\mathrm{~m}, 12 \mathrm{H} \mathrm{Ar}-\mathrm{H}+$ pyridine $\mathrm{H}), 8.30(\mathrm{~s}, 1 \mathrm{H}$, methylenic $\mathrm{H})$ and $11.20\left(\mathrm{~s}, \mathrm{H}, \mathrm{NH}, \mathrm{D}_{2} \mathrm{O}\right.$ exchangeable); $\mathrm{MS}: \mathrm{m} / \mathrm{z}(\%): 512\left(\mathrm{M}^{+}\right.$, $\left.4 \mathrm{Cl}^{35}, 5\right)$. Analysis for $\mathrm{C}_{25} \mathrm{H}_{14} \mathrm{Cl}_{4} \mathrm{~N}_{4}(512.22)$ : required $\mathrm{C}, 58.62 ; \mathrm{H}, 2.75 ; \mathrm{Cl}$, $27.69 ; \mathrm{N}, 10.94$; found $\mathrm{C}, 58.59 ; \mathrm{H}, 2.79 ; \mathrm{Cl}, 27.63 ; \mathrm{N}, 10.90$.

7-(4-Chloro-phenyl)-5-(2, 4-dichloro-phenyl)-3-thioxo-3, 8a-dihydro-[1,2,4] triazolo [4, 3-a] pyridine-8-carbonitrile (19)

To a warmed sodium ethoxide solution $(0.23 \mathrm{gm}$ of sodium metal in $50 \mathrm{ml}$ ethanol, 10 (3.9gm, $0.01 \mathrm{~mole})$ was added. The reaction mixture was heated for $30 \mathrm{~min}$ and the mixture was allowed to cool to room temperature and $10 \mathrm{ml}$ carbon disulfide was added. The reaction mixture was heated under reflux for $8 \mathrm{hr}$, and then poured into water. The formed solid was filtered off, washed with water, dried and recrystallized from methanol to give compound $19.60 \%$ yield ; m.p. $172-174^{\circ} \mathrm{C}$; IR spectrum $\left(\mathrm{KBr}, \mathrm{v}, \mathrm{cm}^{-1}\right)$ : $3126(\mathrm{NH}), 2217(\mathrm{CN}) ;{ }^{1} \mathrm{H}$ NMR spectrum (DMSO-d $\left.\mathrm{d}_{6}, \delta \mathrm{ppm}\right): 6.80(\mathrm{~s}, 1 \mathrm{H}$, pyridine $\mathrm{H}), 7.10-7.40(\mathrm{~m}, 7 \mathrm{H} \mathrm{Ar}-\mathrm{H})$, and 10.50 (s, H, NH, $\mathrm{D}_{2} \mathrm{O}$ exchangeable); $\mathrm{MS}: \mathrm{m} / \mathrm{z}(\%): 432\left(\mathrm{M}^{+}, 3 \mathrm{Cl}^{35}, 60\right)$. Analysis for $\mathrm{C}_{19} \mathrm{H}_{9} \mathrm{Cl}_{3} \mathrm{~N}_{4} \mathrm{~S}$ (431.73): required $\mathrm{C}, 52.86 ; \mathrm{H}, 2.10 ; \mathrm{Cl}, 24.64 ; \mathrm{N}$, 12.98; S, 7.43; found C, 52.80; H, 2.02; Cl, 24.53; N, 13.02; S, 7.49.

4-(4-Chloro- phenyl)- 6-(2,4-dichloro- phenyl)-2- [N'-(2, 3, 4,5,6-pentahydroxyhexylidene)-hydrazino]-nicotinonitrile (20)

A mixture of $10(3.9 \mathrm{gm}, 0.01 \mathrm{~mol})$ and D-glucose $(1.8 \mathrm{gm}, 0.01 \mathrm{~mole}) 50 \mathrm{ml}$ in DMF was heated at $70{ }^{\circ} \mathrm{C}$ for $1 \mathrm{hr}$ in the presence of a catalytic amount of acetic acid. The formed precipitate was filtered off, washed with ethanol and dried to give compound 20 in pure form, $80 \%$ yield ; m.p. $187-189{ }^{\circ} \mathrm{C}$. IR spectrum $\left(\mathrm{KBr}, v, \mathrm{~cm}^{-1}\right): 3460-3100(\mathrm{OH}, \mathrm{NH}), 2215(\mathrm{CN}) ;{ }^{1} \mathrm{H}$ NMR spectrum (DMSO- $\left.\mathrm{d}_{6}, \delta \mathrm{ppm}\right): 3.1-3.36\left(\mathrm{~m}, 2 \mathrm{H}, \mathrm{CH}_{2} \mathrm{OH}\right), 3.38-3.90(\mathrm{~m}, 4 \mathrm{H}$, protons of 
alditol $^{25}$ ), 4.38-4.82 (m, 2H, OH, D $2 \mathrm{O}$ exchangeable), 5.30-5.80 (m, 3H, OH, $\mathrm{D}_{2} \mathrm{O}$ exchangeable $), 6.81(\mathrm{~s}, 1 \mathrm{H}$, pyridine $\mathrm{H}) 7.30-7.94(\mathrm{~m}, 8 \mathrm{H}, \mathrm{Ar} \mathrm{H}+\mathrm{HC}=\mathrm{N})$ and $12.55\left(\mathrm{~s}, 1 \mathrm{H}, \mathrm{NH}, \mathrm{D}_{2} \mathrm{O}\right.$ exchangeable) $\mathrm{MS}: \mathrm{m} / \mathrm{z}(\%): 551\left(\mathrm{M}^{+}, 3 \mathrm{Cl}^{35}, 6\right)$.

\section{References}

1. Amr, A.E., Sayed H.H. and Abdulla, M.A., Synthesis and reactions of some new substituted pyridine and pyrimidine derivatives as analgesic, anticonvulsant and antiparkinsonian agents. Arch. Pharm. Chem. Life Sci. 338, 433-440 (2005).

2. Sayed, H.H., Synthesis and reactions of 3-Aryloxiran-2-yl-pyridin-2-yl methanone. A novel synthesis of triazolopyrimidine for biological evaluation. Egypt. J. Chem. 48 (2), 223-234 (2005).

3. Rashad, A.E., Sayed, H.H. and Shamroukh, A.H., Preparation of some fused pyridopyrimidine and pyridothienotriazine derivatives for biological evaluation, Phosphorus, sulfur and Silicon 180, 2767-2777 (2005).

4. Kumar, S., Das, S., Dey, S., Maity, P., Guha, M., Choubey, V., Panda, G. and Bandyopadhyay, V., Antiplasmodial activity of [(Aryl) arylsulfanylmethyl] pyridine. Antimicrobial Agenta and Chemotherapy, 52 (2),705-715 (2008).

5. Amr, A.E., Mohamed, A.M., Mohamed, S.F., Abdel-Hafez, N. and Hammam, A.G., Anticancer activities of some newly synthesized pyridine, pyrane, and pyrimidine derivatives. Bioorg Med Chem. 14, 5481-5488 (2006).

6. Martin, C., Göggel, R., Dal Piaz, V., Vergelli, C., Giovannoni, P., Ernst, M. and Uhlig, S., Airway relaxant and anti-inflammatory properties of a PDE4 inhibitor with low affinity for the high-affinity rolipram binding site, Naunyn-Schmiedeberg, Arch. of Pharm. 365 (4), 284-289 (2002).

7. Hatanaka, M., Takahashi, K., Nakamura, S. and Mashino, T., Preparation and antioxidant activity of $\alpha$-pyridoin and its derivatives. Bioorg. Med. Chem. 13, 67636770 (2005).

8. Benjahad, A., Oumouch, S., Guillemont, J., Pasquier, E., Mabire, D., Andries, K., Nguyen, C.H. and Grierson, D.S., Structure-activity relationship in the 3-iodo-4phenoxypyridinone (IOPY) series: The nature of the C-3 substituent on anti-HIV activity. Bioorg. Med. Chem. Lett. 17, 712-716 (2007).

9. Zhang, Y., Fan, X., Chakaravarty, D., Xiang, B., Scannevin, R.H., Huang, Z., Ma, J., Burke, S., Karnachi, P., Rhodes, K.J. and Jackson, P.F., 1-Hydroxy-2pyridinone-based MMP inhibitors: Synthesis and biological evaluation for the treatment of ischemic stroke. Bioorg. Med. Chem. Lett. 18, 409-413 (2008).

10. Mukai, A., Nagai1, A., Inaba , S., Takagi, M. and Shin-ya, K., Jbir-, A new 4pyridinone derivative isolated from Penicillium daleae Zaleski fE50, Antibiotics Advance Online Publication 23 October 2009; doi: 10.1038/ja.2009.101.

11. Romero, I.C., Saravia, N.G. and Walker, J., Selective action of fluoroquinolones against intracellular amastigotes of Leishmania (viannia) Panamensis in vitro. J. Parasitology, 91 (6),1474-1479 (2005).

Egypt. J. Chem. 53, No.1 (2010) 
12. Van, K., Cauvin,C., Walque, S., Georges, B., Boland, S., Martinelli,V., Demonte, D., Durant, F., Hevesi, L. and Lint, V.C., New pyridinone derivatives as potent HIV-1 nonnucleoside reverse transcriptase inhibitors. J. Med.Chem. 52 (12), 36363643 (2009).

13. Guba, W., Nettekoven, M., Pullman, B., Riemer, C. and Schmitt, S., Comparison of inhibitory activity of isomeric triazolopyridine derivatives towards adenosine receptor subtypes, Bioorg. \& Med. Chem. Lett. 3307-3312 (2004).

14. Luo, Y. and Hu, Y., Synthesis and antifungal activity of 2-aryl-1,2,4-triazolo[1,5a]pyridine derivatives. Archiv der Pharmazie, 339, 262-266 (2006).

15. Koo, J., New classes of active central nervous system depressing and stimulating agents. J. Org. Chem. 26 (2), 635-636 (1961).

16. Keavtchenko, S. and Largrange, A., PCT. Int. Appl WO. 02 76,416 3 Oct. 2002; Chem. Abst. 137, 268 (2002).

17. Sayed, H.H. and Ali, M.A., Synthesis of 3-[(4-Chloro-phenyl) oxiranyl]thiophen-2yl-propanone and their reactions with some nucleophilles for antiviral evaluations, Phosphorus, Sulfur and Silicon, 183, 156-167 (2008).

18. Yun, M.L., Cheng, M.S., Wei, F., Lin, R.C., But, P.P., Lee, H.S. and Lee, S.F., Uncinoside A and B, two new antiviral chromone glycosides from Selaginella uncinata. Chem. Pharm. Bull. 51(11), 1264-1267 (2003).

19. Desideri, N., Mastromarino, P. and Conti, C., Synthesis and evaluation of antirhinovirus activity of 3-hydroxy and 3-methoxy 2-styrylchromones. Antiviral Chemistry \& Chemotherapy, 14, 195-203 (2003).

20. Furuichi, K., Takashi, W., Iwata, Y., Sukaia, N., Yoshimoto, K., Kobayashi, K., Mukaida, N., Matsushima, K. and Yokoyama, H., Administration of F R167653, A new anti-inflamatory compound prevents renal ischaemial/ reperfusion injury in mice. Nephrol Dial Transphant, 17, 399-407 (2002).

21. Kannan, S., Balakrishnam, B., Muzik, O., Romero, R. and Chugani, D., Positron emission tomography imaging of neuroinflammation, Jornal of Child Neurology, 24 (9), 1190-1199 (2009).

22. El-Emary, T.I., Al-Muaikel, N. and Moustafa, O.S., Synthesis and antimicrobial activity of some new heterocycles based on 3-Methyl-1-phenyl-5-benzene sulfonamido pyrazole. Phosphorus, Sulfur and Silicon, Relat. Elem. 177, 195-210 (2002).

23. Salama, M.A., Sayed, G.H., Abdou, W.A.M., Maghraby, A.S. and Flefel, E.M., Synthesis and immunological studies of some new substituted tetrahydronaphthalene on biomphalaria Alexandrena snails. Bull. Fac. Pharm. Cairo Univ. 35, 65-71 (1996).

24. Rashad, A.E. and Ali, M.A., Synthesis and antiviral screening of some thieno[2,3d]pyrimidine nucleosides. Nucleos, Nucleot and Nucleic Acids, 25, 17-28 (2006). 
25. Rashad, A.E., Shamroukh, A.H., Ali, M.A. and AbdelMotti, F.M., Synthesis and antiviral screening of some novel pyridazines and triazolopyridazine nucleosides. Heteroatom Chem. 18, 274-282 (2007).

26. Bauer, A.W., Kirby, M.M., Sherris, J.C. and Turck, M., Antibiotic susceptibility testing by a standardizwd single disc method. Am. J. Clin. Pahol. 45,493-496 (1996).

27. Lui, H.R. and John, F., Potintial; cell culture models for anti oxidant research. $J$. Agric. Food Chem. 53 (10), 4311-4314 (2005).

28. Halliwell, B. and Gutteridge, J.M.C., In: Free Radical in Biology \& Medicine. $2^{\text {nd }}$ ed ., Oxford: Clarenson, 416-508 (1989).

29. Kanner, J., Oxidative process in meat \& meat product, quality implications. J. Meat Science, 36, 169-189 (1994).

30. Hudson, B.J.F., The Mechanism of Antioxidant Action In vitro (ed), Food Antioxidants. London: Elsevier Applied Science (1990).

31. Inatani, R., Nakatani, N. and Fuwa, H., Antioxidative effect of the contituents of rosemary ( Rosmarinus officinalts L.). Agric. Biol. Chem. 47, 521-528 (1983).

32. Hesse, M., Meier, H. and Zee, B., Spektroskopische methoden in der organischen chemie. Georg Thieme Verlag Stuttgart, P.391 (1979).

( Received 11/1/2010; accepted 16/2/2010 ) 


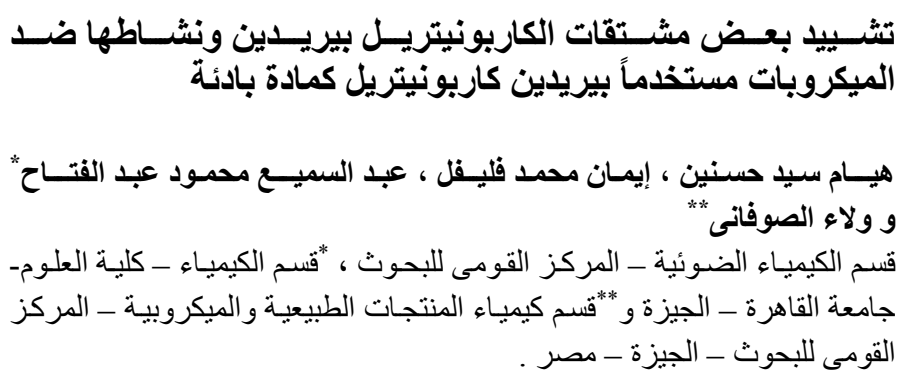

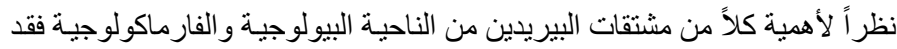

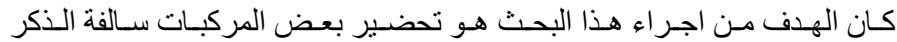

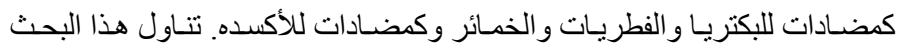

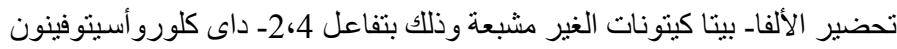

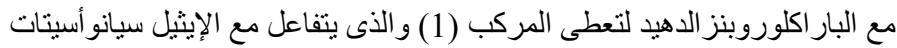

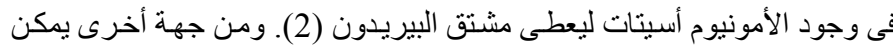

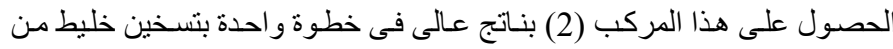

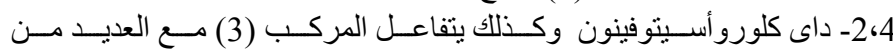

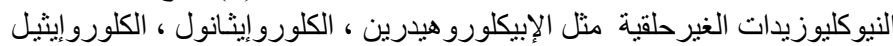

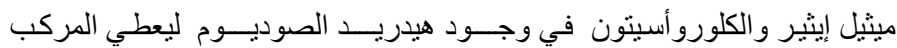

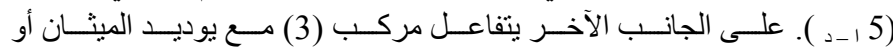

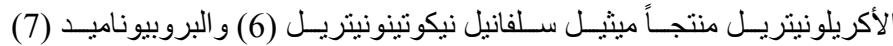

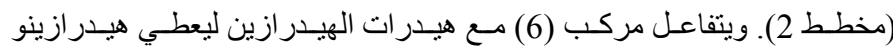

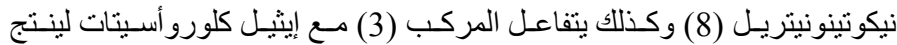

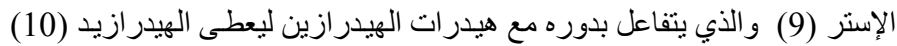

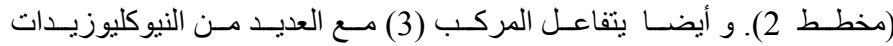

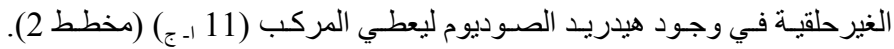

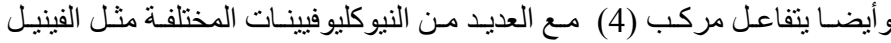
هيدرازين، الأنيلين، الجليسين ليعطى المركبات (12 - 14) علئ النيل التو الى (مخطط

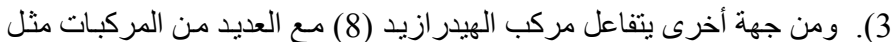

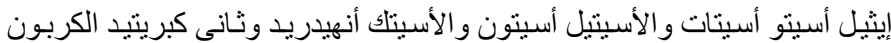

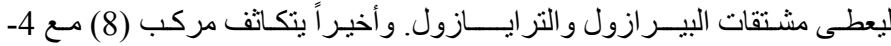

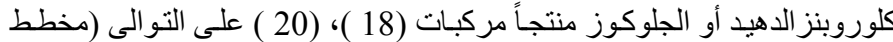

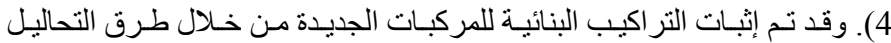

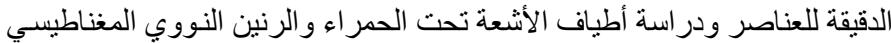

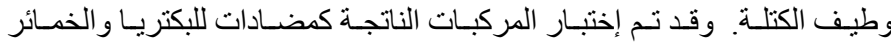
و الفطريات وكذلك كمضادات للأكسدة. 